\title{
Surgical Treatment of Coronary to Pulmonary Artery Fistulas in Adults
}

\author{
Yangsin Lee, ${ }^{1} \mathrm{MD}$, Yoshihiro Naruse, ${ }^{1} \mathrm{PhD}$ and Keita Tanaka, ${ }^{1} \mathrm{PhD}$
}

\begin{abstract}
Summary
Coronary to pulmonary artery fistulas (CPAFs) are abnormal communications between the coronary and pulmonary arteries. They are an uncommon congenital heart disease and usually remain asymptomatic until later in life. However, there is no consensus on their management. We present four adult patients who required surgery for coronary to pulmonary artery fistulas to illuminate this issue. The clinical presentations were variable depending on the anatomical features of coronary to pulmonary artery fistulas and the presence or absence of other cardiac diseases. We successfully performed surgical closure of the coronary to pulmonary artery fistulas in each of the cases. In this report, we describe our experience with these cases and outline the available therapeutic strategies and treatment options for coronary to pulmonary artery fistulas.
\end{abstract}

(Int Heart J 2017; 58: 1012-1016)

Key words: Cardiac surgery, Coronary artery fistula, Coronary artery anomaly, Coronary aneurysm

A coronary artery fistula (CAF) is an abnormal communication between a coronary artery and either a cardiac chamber or a great vessel including pulmonary artery (PA). The reported incidence is $0.002 \%$ among the general population, ${ }^{1)}$ and these fistulas account for approximately $0.4 \%$ of all congenital cardiac abnormalities. ${ }^{2}$ Though they usually remain asymptomatic in childhood, they may lead to congestive heart failure, myocardial ischemia, infective endocarditis, or rupture of an associated aneurysm later in life. ${ }^{3)}$ Recently, multidetector computed tomography (MDCT) has been used to delineate the complex anatomy of $\mathrm{CAF}^{4)}$ or coronary aneurysm, ${ }^{5}$ with recent studies indicating that coronary to pulmonary artery fistulas (CPAFs) are the most common type ${ }^{6-8)}$ It has been argued that the embryological basis of CPAF is different from other types of CAF, which drain into cardiac chambers, ${ }^{9,10)}$ and that the resulting left-toright shunt is relatively small through a CPAF. ${ }^{11)}$ To date, the optimal management for small and asymptomatic CPAFs remains a matter of debate. In this report, we present our experience with surgical repair of CPAF at our institution between January 2001 and June 2016.

\section{Case Reports}

Case 1: An asymptomatic 70-year-old woman with a history of hypertension and hyperlipidemia was referred to our hospital for evaluation after a mass adjoining the left cardiac border had been identified on chest X-ray. En- hanced CT of the chest demonstrated a large aneurysm measuring $34 \mathrm{~mm}$ in diameter on the left side of the main PA (Figure 1A). The aneurysm had increased in size by 6 $\mathrm{mm}$ over a 3-year period. Coronary angiography (CAG) revealed a fistula arising from diagonal branch that was supplying a large aneurysm draining into the PA through two fistulas (Figure 1B). Electrocardiography (ECG) showed no ischemic changes, and echocardiography revealed preserved left ventricular systolic function with no significant valvular disease. From these findings, she was diagnosed as having a CPAF with a concomitant large aneurysm that was considered to have enlarged progressively. Surgical resection was then chosen and performed under cardiac arrest and cardiopulmonary bypass (CPB). The aneurysm was opened, and the two small orifices were noted before being closed with pledgetted sutures from the inside. The afferent and efferent vessels were then ligated and the aneurysm was resected. Pathophysiological study showed that the aneurysm was a true aneurysm, and the structure of the media had mostly disappeared, which resulted in arterial wall thinning. The intima was thickened by fibrous tissue layers with calcific lesions, thrombus, and macrophage foam cells, suggesting atherosclerotic changes. ${ }^{12)}$ The patient recovered well postoperatively, and his condition remained stable 7 years after the operation.

Case 2: A 77-year-old man with a history of hypertension was referred to our hospital with exertional chest pain and syncope. His ECG showed ST depression in leads V5 and

From the ${ }^{1}$ Department of Cardiovascular Surgery, Cardiovascular Center, Toranomon Hospital, Tokyo, Japan.

Address for correspondence: Yangsin Lee, MD, Department of Cardiovascular Surgery, Cardiovascular Center, Toranomon Hospital, 2-2-2 Toranomon, Minato-ku, Tokyo 105-8470, Japan. E-mail: o9o19810704@gmail.com

Received for publication December 15, 2016. Revised and accepted February 14, 2017.

Released in advance online on J-STAGE November 17, 2017.

doi: 10.1536/ihj.16-627

All rights reserved by the International Heart Journal Association. 

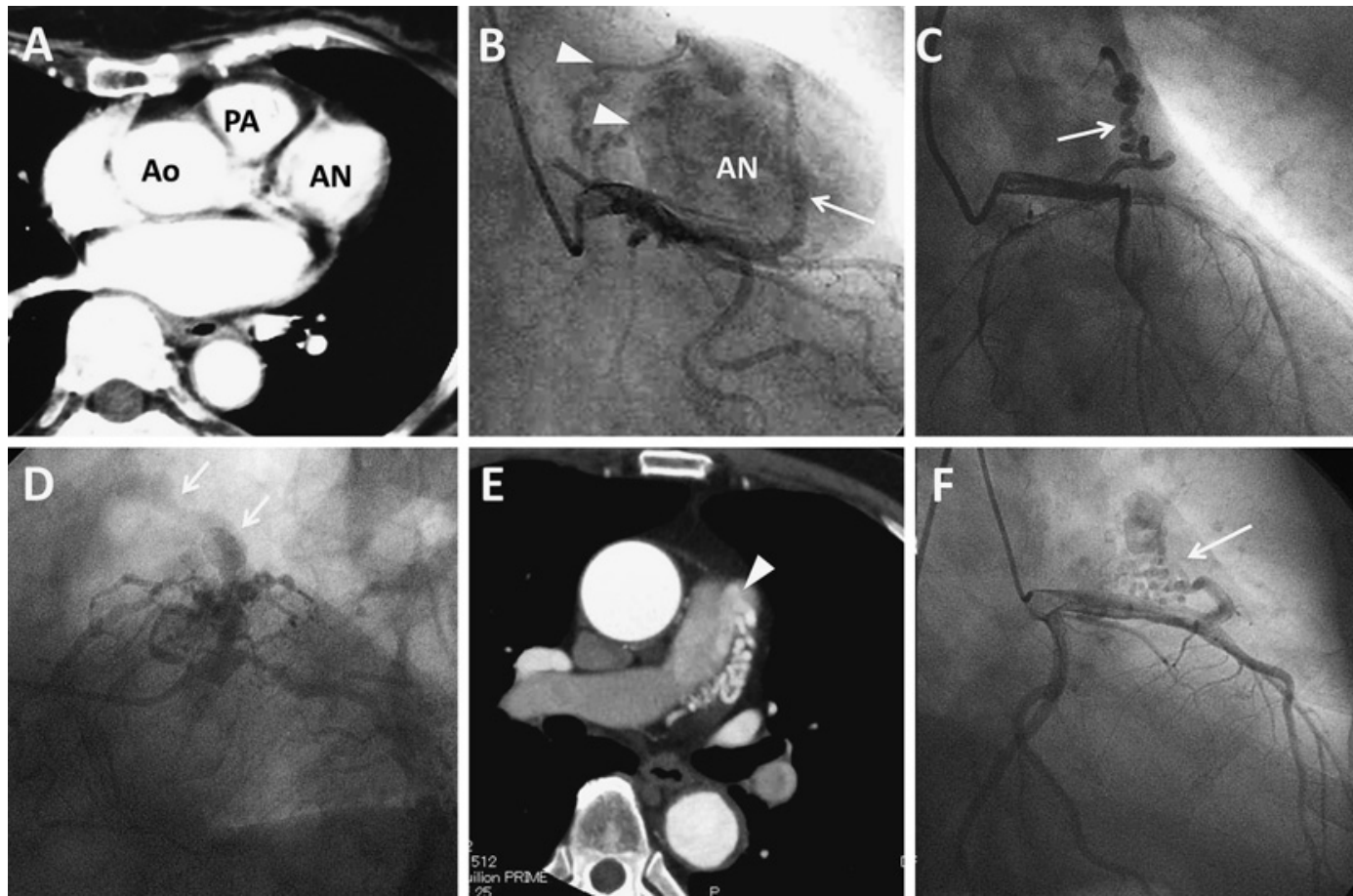

Figure 1. Computed tomography showing the following. A: a large aneurysm located to the left of the main PA (Case 1) and E: tortuous vessels around the main pulmonary artery with a high-density jet flow (arrowhead) directly into the main PA (Case 3). Coronary angiography showing the following. B: a fistula originating from the diagonal branch supplying a large aneurysm (arrow) with the aneurysm draining into the PA through two fistulas (arrowheads) (Case 1); C: a tortuous fistula originating from the left main trunk and directly entering the main PA (arrow) (Case 2); D: a dilated and tortuous fistula originating from the proximal left anterior descending artery (arrows) (Case 3); and F: a twisting fistula originating from the left anterior descending artery and draining into the main PA (arrow) (Case 4). AN, aneurysm; Ao, ascending aorta; PA, pulmonary artery.

V6, and transthoracic echocardiography confirmed severe aortic valve stenosis with preserved left ventricular ejection fraction (LVEF) of $69 \%$. A single fistula, originating from the left main trunk and draining into the main PA, was identified by CAG (Figure 1C). He therefore underwent surgery via median sternotomy, and the thin fistula was confirmed on the anterior side of the main PA. Under $\mathrm{CPB}$, the fistula was ligated proximal to the PA, before inducing cardiac arrest with cold crystalloid cardioplegia to perform aortic valve replacement. The postoperative course was uneventful, and his condition remained stable 9 years after surgery.

Case 3: An asymptomatic 74-year-old man was referred for investigation of possible ischemic heart disease before surgery for benign prostatic hyperplasia. In the Master double-step test, the ECG showed ST depression in leads II, III, aVF, and V3-V6. Echocardiography revealed normal left ventricular contractility with mild aortic, mitral, and tricuspid regurgitation. CAG also revealed 90\% stenosis of the left anterior descending (LAD) artery (\#6-7), $75 \%$ stenosis of the proximal segment of the diagonal branch, $75 \%$ diffuse stenosis of the proximal segment of the circumflex coronary artery, and total occlusion of the right coronary artery (nondominant). A grossly dilated and tortuous CAF was found arising from the most proximal segment of the LAD, proximal to the LAD stenosis, entering the PA (Figure 1D). MDCT confirmed an anomalous tortuous vessel arising from the proximal LAD (Figure 1
E). The patient underwent surgery via median sternotomy under cardiac arrest and CPB. The main PA was opened and the entry point of the fistula was noted to be $5 \mathrm{~mm}$ in diameter and then closed with pledgetted sutures. Epicardial ligations were added to the dilated and tortuous fistula in the anterior aspect of the PA, and we performed three-vessel coronary artery bypass grafting: left internal mammary artery to LAD, saphenous vein graft to left posterolateral artery, and gastroepiploic artery to left posterior descending artery. The patient made an uneventful postoperative recovery and was symptom-free at follow-up 3 years later.

Case 4: A 47-year-old man receiving dialysis presented with chest pain at rest and attended via ambulance. He had a surgical arteriovenous fistula formed in his left forearm when he was 45 years old. The admission ECG showed new-onset negative T-waves in leads II, III, and aVF. Echocardiography showed a moderately impaired LVEF of $41 \%$, left ventricular dilation, and mild mitral regurgitation. CAG showed a fistula between the LAD artery and the PA (Figure 1F), with no evidence of significant coronary stenosis. However, right heart catheterization uncovered a cardiac output of $9.9 \mathrm{~L} / \mathrm{min}$ and a cardiac index of $5.3 \mathrm{~L} / \mathrm{min} / \mathrm{m}^{2}$, indicating high-output congestive heart failure. The pulmonic-to-systemic flow ratio (Qp/Qs) was 1.37. MDCT revealed a network of fistulas between the LAD and the separately branched conus artery forming a small aneurysm in the anterior aspect of 

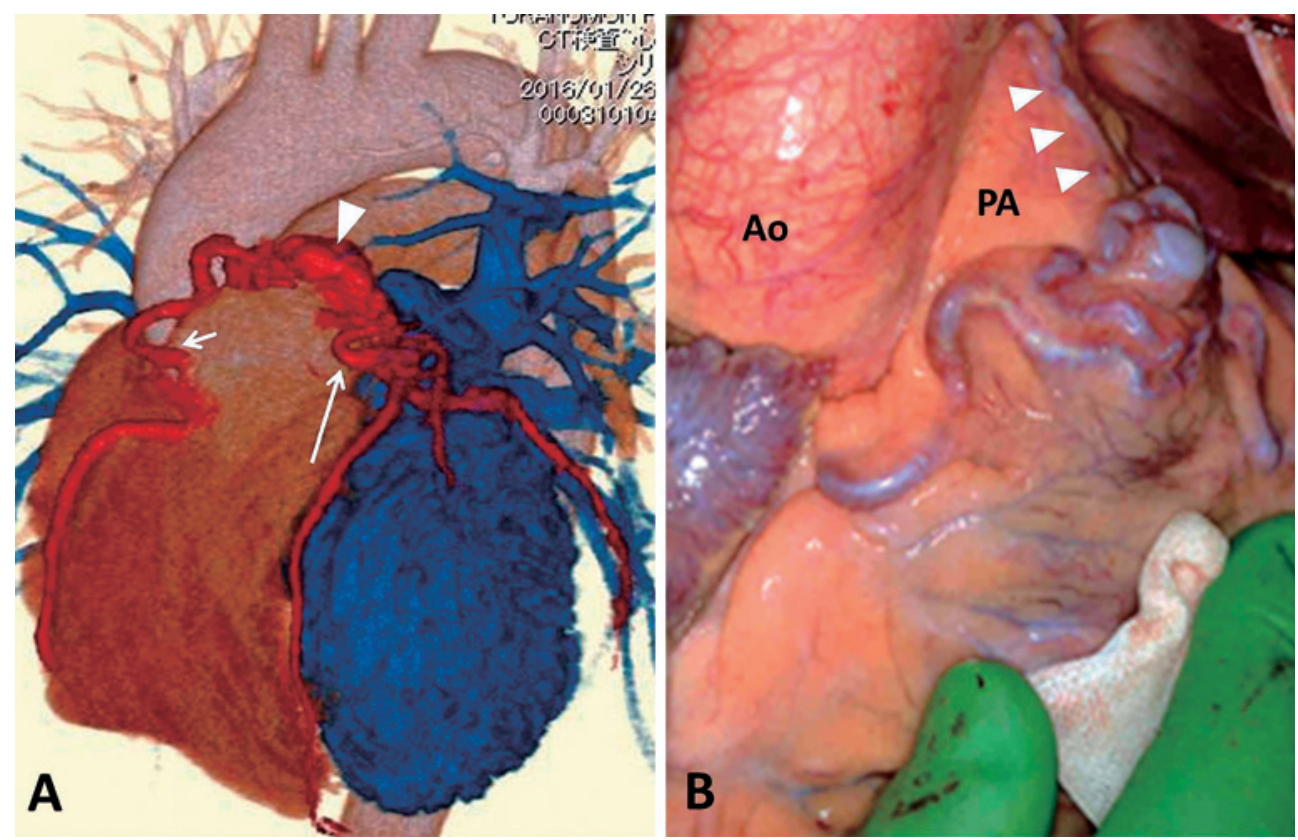

Figure 2. A: Multidetector computed tomography showing multiple fistulas originating from both the left anterior descending artery (long arrow) and the separately branching conus artery (short arrow), together with an associated aneurysm (arrowhead). B: The intraoperative photograph shows two abnormal vessels running down from the systemic artery (arrowheads), supplying the coronary to pulmonary artery fistula. Ao, ascending aorta; PA, pulmonary artery.

the PA (Figure 2A). The flow rate in the arteriovenous dialysis shunt was within normal limits $(650 \mathrm{~mL} / \mathrm{min})$. The patient underwent surgery by median sternotomy under cardiac arrest and CPB. Intraoperatively, we identified the two small fistulas running down from the systemic artery to the main PA (Figure 2B). The main PA was then opened longitudinally, and a single efferent hole measuring $3 \mathrm{~mm}$ in diameter, located in the left lateral aspect of the pulmonary trunk, was closed with pledgetted sutures. Epicardial ligations were added to the afferent vessels coming from the LAD, conus, and systemic arteries, and the aneurysmal sac was ligated. A postoperative CT showed no residual fistulas and that the LVEF had improved to $61 \%$. The patient was symptom-free at 6 months after surgery.

\section{Discussion}

The findings of our case report are consistent with the known morphology of CPAFs, which can be classified into anterior (pre-truncal) and posterior (retro-truncal) types. ${ }^{13)}$ Most CPAFs are asymptomatic anterior types, which usually arise from the proximal left and/or right coronary arteries via the anterior conus branches and connect to the anterior wall of the main PA. ${ }^{13)}$ All of our cases had anterior CPAFs. In a recent large cohort based on MDCT data, CPAFs arising from both coronary arteries (e.g., Case 4 in this report) were reported in approximately $50 \%$ of cases. ${ }^{6-8)}$ By contrast, posterior CPAFs arise via small atrial branches directly from the left circumflex or left sinoatrial node artery and connect to the right PA through the transverse sinus. ${ }^{13)}$ Although extracar- diac vascular supply from the systemic circulation (e.g., the bronchial artery, left subclavian artery, or internal mammary artery) is more common in posterior types, it occurs in $5 \%-10 \%$ of anterior types. ${ }^{6,7)}$ Aneurysmal sac formation is associated with CPAFs in $15 \%-35 \%$ of cases. ${ }^{6-8)}$

Most CPAFs occur as isolated lesions, but they may also be associated with other congenital or acquired cardiac lesions, such as patent ductus arteriosus, ventricular septal defect, atrial septal defect, valve disease, or coronary disease. ${ }^{14,15)}$ In these cases, fistulas can potentiate the symptoms of the other pathology. ${ }^{9}$ ) Therefore, it would be reasonable to consider CPAFs as good candidates for concomitant surgery if they are associated with cardiovascular lesions that need repair.

There is general agreement that symptomatic patients should undergo corrective surgery. It should be noted, however, that symptoms caused by an isolated CAF are not directly related to shunt size; rather, they are related to the presence of long-standing modest left-to-right shunts. ${ }^{16)}$ Given this, special attention should perhaps be given to patients with CPAFs who are receiving hemodialysis. As occurred in Case 4, the arteriovenous shunt created for hemodialysis, coupled with the already existing left-to-right shunt due to the CPAF, caused heart failure to develop. In patients with CPAF, the additional arteriovenous shunt required for hemodialysis may hasten symptom onset.

In contrast to these examples, the optimal management for asymptomatic patients remains less clear. Some authors have reported that asymptomatic patients with small CAFs do not require immediate treatment and can 
be managed with regular follow-up alone. ${ }^{17)}$ Aneurysm formation, even in asymptomatic patients, should, however, be considered an indication for surgery because of the potential for unexpected rupture. Some studies have reported that aneurysms larger than $3 \mathrm{~cm}$ in diameter should be considered for surgery. ${ }^{18)}$ However, even small saccular aneurysms measuring $1 \mathrm{~cm}$ in diameter can cause cardiac tamponade if they rupture, concluding that aneurysms associated with CPAFs should be resected regardless of the size. ${ }^{19)}$

Two main treatment options are available when presented with CPAF: transcatheter or surgical closure. Surgical treatment is recommended for extremely tortuous, multiple, or aneurysmal fistulas, as well as for fistulas associated with concomitant cardiac disease requiring surgery. ${ }^{20)}$ Surgical techniques include direct epicardial ligation, internal closure of the fistula within the PA, and aneurysm excision. These surgical techniques should be applied according to the morphology of CPAFs. For CPAFs with a single communication, direct epicardial ligation of the fistula can be performed in the beating heart. When CPAFs are simultaneously operated with associated concomitant cardiac diseases, this method is effective in terms of myocardial protection, without increasing the surgical invasion. As presented in Case 2, we ligated the fistula before inducing cardiac arrest because the fistula might cause runoff into the PA leading to inadequate myocardial protection during administration of cardioplegia. Meanwhile, when the fistula forms a large aneurysm, the excision of the aneurysm is necessary because leaving the aneurysm is associated with the risk of rupture later. The aneurysm should be opened and the orifices can be closed from the inside, in addition to epicardial ligations of the afferent and efferent vessels. As presented in Case 1, we could safely exclude the aneurysm without compromising the coronary artery. Pulmonary arteriotomy and internal closure of the efferent site of CPAFs might be best suited for large tortuous or multiple CPAFs as presented in cases 3 and 4. Furthermore, transpulmonary closure, with subsequent epicardial ligation of all the afferent vessels, is the most certain way to completely obliterate the shunt.

To date, there is no consensus on medical management after surgical closure of CAF. Most studies do not suggest antiplatelet or anticoagulation treatment. ${ }^{1)}$ However, some authors advocate antiplatelet treatment with aspirin $^{21)}$ and anticoagulation treatment with warfarin ${ }^{22)}$ in patients with a dilated residual native coronary artery proximal to the fistula to prevent coronary ischemia and thromboembolic events. In our case, warfarin was administered to prevent valve thrombosis after aortic valve replacement (Case 2) and aspirin to improve the graft patency after coronary artery bypass grafting (Case 3). Meanwhile, no antithrombotic medication was administered to cases 1 and 4 because their principal coronary arteries were not dilated.

\section{Conclusion}

We reported the details of four adults with CPAF who presented diverse pathological conditions and under- went surgical correction. On the basis of these case reports and the subsequent review, we conclude that the optimal management strategy for CPAF depends on the presence of symptoms, the anatomical features of a given fistula (including aneurysm formation), and the presence of other cardiovascular diseases. However, in all cases, treatment must be individualized to each patient.

\section{Disclosures}

Conflicts of interest: The authors have no potential conflicts of interest or funding source to report.

\section{References}

1. Luo L, Kebede S, Wu S, Stouffer GA. Coronary artery fistulae. Am J Med Sci 2006; 332: 79-84 (Review).

2. Burch GH, Sahn DJ. Congenital coronary artery anomalies: the pediatric perspective. Coron Artery Dis 2001; 12: 605-16 (Review).

3. Liberthson RR, Sagar K, Berkoben JP, Weintraub RM, Levine FH. Congenital coronary arteriovenous fistula. Report of 13 patients, review of the literature and delineation of management. Circulation 1979; 59: 849-54.

4. Niwa R, Hasumi E, Fujiu K, et al. A case of multiple coronary artery-left ventricular micro fistulae complicated with hepatic arteriovenous fistulae. Int Heart J 2016; 57: 123-6.

5. Motozawa Y, Uozumi H, Maemura S, et al. Acute myocardial infarction that resulted from poor adherence to medical treatment for giant coronary aneurysm. Int Heart J 2015; 56: 551-4.

6. Zhang LJ, Zhou CS, Wang Y, et al. Prevalence and types of coronary to pulmonary artery fistula in a Chinese population at dual-source CT coronary angiography. Acta Radiol 2014; 55: 1031-9.

7. Lim JJ, Jung JI, Lee BY, Lee HG. Prevalence and types of coronary artery fistulas detected with coronary CT angiography. AJR Am J Roentgenol 2014; 203: W237-43.

8. Kim MS, Jung JI, Chun HJ. Coronary to pulmonary artery fistula: morphologic features at multidetector CT. Int J Cardiovasc Imaging 2010; 26: 273-80.

9. Baim DS, Kline H, Silverman JF. Bilateral coronary arterypulmonary artery fistulas. Report of five cases and review of the literature. Circulation 1982; 65: 810-5.

10. Vaidyanathan KR, Theodore SA, Sankar MN, Cherian KM. Coronary artery to pulmonary artery fistula with dual originembryological, clinical and surgical significance. Eur J Cardiothorac Surg 2007; 31: 318-9.

11. Gowda RM, Vasavada BC, Khan IA. Coronary artery fistulas: clinical and therapeutic considerations. Int J Cardiol 2006; 107: 7-10 (Review).

12. Soeki T, Sata M. Inflammatory biomarkers and atherosclerosis. Int Heart J 2016; 57: 134-9 (Review).

13. Saremi F, Patel A, Shavelle D, Licht JR, Kang J. Left circumflex coronary-pulmonary artery fistula and transmediastinal participation of bronchial arteries best shown by CT. Int J Cardiol 2014; 177: e120-4.

14. Bogers AJ, Quaegebeur JM, Huysmans HA. Early and late results of surgical treatment of congenital coronary artery fistula. Thorax 1987; 42: 369-73.

15. Kamiya H, Yasuda T, Nagamine H, et al. Surgical treatment of congenital coronary artery fistulas: 27 years' experience and a review of the literature. J Card Surg 2002; 17: 173-7 (Review).

16. Sunder KR, Balakrishnan KG, Tharakan JA, et al. Coronary artery fistula in children and adults: a review of 25 cases with long-term observations. Int J Cardiol 1997; 58: 47-53.

17. Challoumas D, Pericleous A, Dimitrakaki IA, Danelatos C, 
Dimitrakakis G. Coronary arteriovenous fistulae: a review. Int J Angiol 2014; 23: 1-10 (Review).

18. Izumi K, Hisata Y, Hazam S. Surgical repair for a coronarypulmonary artery fistula with a saccular aneurysm of the coronary artery. Ann Thorac Cardiovasc Surg 2009; 15: 194-7.

19. Sakao T, Tsunooka N, Nakagawa H, Kajiwara S. Ruptured saccular aneurysm of a coronary artery to pulmonary artery fistula associated with cardiac tamponade. Kyobu Geka 2000; 53: 6846(Japanese).
20. Mangukia CV. Coronary artery fistula. Ann Thorac Surg 2012; 93: 2084-92 (Review).

21. Hou B, Ma WG, Zhang J, et al. Surgical management of left circumflex coronary artery fistula: a 25 -year single-center experience in 29 patients. Ann Thorac Surg 2014; 97: 530-6.

22. Gowda ST, Latson LA, Kutty S, Prieto LR. Intermediate to long-term outcome following congenital coronary artery fistulae closure with focus on thrombus formation. Am J Cardiol 2011; 107: 302-8. 